\title{
RESEARCH ON THE NON-LINEAR SPINOR THEORY WITH INDEFINITE METRIC IN HILBERT SPACE
}

\author{
W. HEISENBERG \\ Max-Planck Institut für Physik, Göttingen
}

The present report will be confined to recent developments in a non-linear spinor theory of matter, that had been treated in several previous papers ${ }^{1)}$. The theory is different from the conventional theories especially by the use of an indefinite metric in Hilbert space and by the introduction of dipole-ghost states, the rôle of which has been analysed in some detail with the help of the Lee model $^{2)}$.

The theory in the form that has been suggested ${ }^{3)}$ in connection with Pauli's transformation ${ }^{4)}$ starts from two characteristic invariants :

$J=\int \bar{\psi} \gamma_{\nu} \frac{\partial}{\partial x_{\nu}} \psi d \tau \quad$ and $\quad I=\int\left(\dot{\psi} \gamma_{\mu} \gamma_{5} \psi\right)^{2} d \tau$

Both expressions are invariant with respect to the Lorentz group, the Pauli - Gürsey group ${ }^{5)}$ and the PCT-transformations; $J$ is the conventional Lagrangian of the neutrino theory.

If one chooses any function of $J$ and $I$ as Lagrangian, the variation of $\mathfrak{L}: \delta \mathfrak{L}=0$ leads to the wave equation:

$$
\gamma_{\mu} \frac{\partial}{\partial x_{\mu}} \psi \pm \ell^{2} \gamma_{\mu} \gamma_{5} \psi\left(\bar{\psi} \gamma_{\mu} \gamma_{5} \psi\right)=0
$$

(The constant $\frac{\partial \mathfrak{I}}{\partial I} / \frac{\partial \mathfrak{L}}{\partial J}$ has the dimension of a square of a length and may be put equal to $\pm \ell^{2}$.)

The determination of some of the simplest mass eigenvalues has been carried out recently by Mitter and Schlieder ${ }^{(*)}$ in a first approximation by means of the Tamm Dancoff method. This method seems for the time being to be the only method available for treating problems of a relativistic non-linear field theory with indefinite metric, in which the commutator cannot be given from the beginning but must come out as a result of the regularity conditions. While the mathematical basis of a non-linear spinor theory with indefinite metric is at present still uncertain in a similar manner as the more conventional theories involving interactions, there seems to be no reason to consider the Tamm - Dancoff method (when applied to a mathematically well-defined eigenvalue problem) as less reliable than other approximation methods.

The Tamm - Dancoff method needs to begin with an assumption about the form of the commutator, the consistency of which has to be checked later in the course of the calculations. This process has been described in detail in some of the previous papers ${ }^{6)}$. In a first approximation it is convenient to restrict the calculation to the "strong interaction" terms where one has full invariance for the isospin group, and to look apart from such complications as -conjugation and degeneration of the vacuum discussed in the preprint ${ }^{3}$.

The only assumption for the commutator showing the complete isospin invariance seems to be

$$
\begin{aligned}
\left\langle\psi_{\alpha}(x) \bar{\psi}_{\beta}\left(x^{\prime}\right)\right\rangle & =-\gamma_{\nu}^{\alpha \beta} \frac{\partial}{\partial x_{v}} F(s) \\
& =2 \int e^{i p\left(x-x^{\prime}\right)} d p d K \frac{p_{\nu} \gamma_{\nu}^{\alpha \beta}}{p^{2}+K^{2}} \cdot \varrho(K) \\
& \approx 2 \int e^{i p\left(x-x^{\prime}\right)} d p\left[\frac{p_{\nu} \gamma_{\nu}^{\alpha \beta}}{p^{2}+K^{2}}-\frac{p_{\nu} \gamma_{\nu}^{\alpha \beta}}{p^{2}}+\frac{p_{\nu} \gamma_{\nu}^{\alpha \beta} \cdot K^{2}}{\left(p^{2}\right)^{2}}\right] .
\end{aligned}
$$

In the last line, which is meant as a first approximation, only one discrete mass eigenvalue is considered; the last two terms represent the contributions from the "dipoleghost". Other discrete eigenvalues and the continuous spectrum have been neglected.

If (3) is used as a "contraction function" in the Tamm Dancoff method, the graph $(\uparrow$ leads for a spinor particle of momentum vector $\mathfrak{I}_{\mu}$ to an eigenvalue equation of the form

$$
f\left(\mathfrak{I}^{2}\right) \cdot \mathfrak{I}_{\mu} \gamma_{\mu}{ }^{\alpha \beta} \varphi_{\beta}=0 .
$$

The function $f\left(\mathfrak{I}^{2}\right)$ has been determined numerically by Mitter and Schlieder and has only one zero defining a mass value ${ }^{6)}$.

$\left(^{*}\right)$ A detailed account of these calculations and other discussions connected with the non-linear spinor theory will be published by the Göttingen group in the Zeitschr. f. Naturforschung. 
The solutions of (4) can be divided into two groups. The one belongs to the Dirac equation $\mathfrak{\Im}_{v} \gamma_{\nu} \varphi=0$ and the mass value zero. It can be interpreted as defining four two-component leptons of mass zero $\left(e^{+}, e^{-}, v \bar{v}\right)$.

The other group belongs to a Klein - Gordon equation for a spinor wave function of the type :

$$
\left(\mathfrak{I}_{\mu}^{2}+K^{2}\right) \varphi_{\alpha}=0
$$

It describes four baryons of equal mass $(P N \bar{P} \bar{N})$. Equation (5) can be decomposed into two Dirac equations

$$
\begin{aligned}
& \mathfrak{I}_{\mu} \gamma_{\mu} \varphi=i K \hat{\varphi}, \\
& \mathfrak{I}_{\mu} \gamma_{\mu} \hat{\varphi}=i K \varphi,
\end{aligned}
$$

as had been shown earlier by Gürsey. The symmetry between the matrix elements ( $\varphi$-functions) $\varphi$ and $\hat{\varphi}$ suggests the introduction of the ${ }^{\wedge}$-conjugation and the use of a spinor

$$
\Psi=\left(\begin{array}{c}
\psi \\
\hat{\psi}
\end{array}\right)
$$

If one introduces Pauli-matrices $\Sigma$ in this spinor space, one may write $\widehat{\psi}=\Sigma_{1} \psi$ and replace (5) by

$$
\left(\mathfrak{J}_{\mu} \gamma_{\mu}-i K \Sigma_{1}\right) \varphi=0 .
$$

The group theoretical problems connected with the ${ }^{\wedge}$-conjugation and the reformulation of the commutator with the help of the $\Sigma$-matrices have been treated in detail by Duerr. We mention as one of the results the form of the Pauli-transformations, which must - as one sees from (6) - be written as

$$
\begin{aligned}
& \Psi \rightarrow a \Psi+b \gamma_{5} \Sigma_{3} C^{-1} \bar{\Psi} \\
& \Psi \rightarrow e^{i \alpha \gamma_{5} \Sigma_{3}} \Psi
\end{aligned}
$$

Before coming to the symmetry properties of the solutions we have to discuss the three transformations $\mathrm{P}, \mathrm{C}, \mathrm{T}$ (parity, charge conjugation and time reversal). Since the Lagrangian is invariant for these transformations one would expect corresponding quantum numbers. In the present theory, however, neither $P$ nor $C$ commute with the Pauli-transformations describing charge and baryonic number, if $P$ is written in the conventional form

$$
P: \quad x_{k} \rightarrow-x_{k} \quad, \quad \Psi \rightarrow \gamma_{4} \Psi
$$

Therefore, this kind of parity cannot be defined for particles having a definite baryonic number; the only exception being particles with baryonic number zero, e.g. $\pi$ mesons, since their wave function is not affected by the transformation $e^{i a \gamma_{5}}$.

One may, however, define a second kind of parity by the relation

$$
P: \quad x_{k} \rightarrow-x_{k}, \quad \Psi \rightarrow \gamma_{4} \Sigma_{1} \Psi .
$$

This operation commutes with the Pauli-transformations, since $\gamma_{4} \Sigma_{1}$ commutes with $\gamma_{5} \Sigma_{3}$. Therefore, one can define a parity for heavy particles by means of (11) and it is this second kind of parity that can be used for deriving selection rules. At this point it may be added that in the present theory the transition from particle to antiparticle is not given by the operation $C$, but by $P C$ (first kind of $P$ ) or by $C \Sigma_{1}$.

Similar calculations as for the nucleons have been carried out by Mitter and Schlieder for the $\pi$ meson group, i.e. for particles of baryonic number zero. The method used was in both cases indentical with the method applied in ${ }^{6)}$. The calculations have so far been restricted to particles of spin zero. In this first approximation which is defined by the graph $($, the integral equation for the eigenvalues refers to eigenfunctions in which the two particles are at the same point. This restriction means that one has to do only with $s$-states. For particles of spin 1 one could scarcely hope to get an approximation without taking the $p$-states into account.

Table I gives the representation of the different particles in the form of the operators which annihilate $(A)$ or create $(C)$ the particles in question. If one wants to construct operators that only annihilate or only create, one has to combine the $\psi$ - and the $\hat{\psi}$-term. (The explanation of the signs is given at the bottom of the table.)

Since, on account of the complexity of the calculations, it has so far not been possible to go to a higher approximation in the Tamm - Dancoff method, i.e. to an approximation defined by a more complicated graph, one may try to get some check on the reliability of the calculations by making use of the $\hat{\psi}$-functions. The symmetry between the $\psi$ - and the $\hat{\psi}$-function suggests that one might get a better approximation, when one replaces $\bar{\psi} \gamma_{\mu} \gamma_{5} \psi$ in the calculations by $\frac{1}{2}\left(\bar{\psi} \gamma_{\mu} \gamma_{5} \psi+\hat{\bar{\psi}} \gamma_{\mu} \gamma_{5} \hat{\psi}\right)$. The numbers given in Table II as second approximation refer to this procedure. The parity of the neutron comes out as opposite to the parity of the proton, while the charged pions become scalar particles. This result is not identical with the usual description in the text books (where proton and neutron have the same parity and the $\pi^{ \pm}$particle is pseudoscalar), but it is equivalent to the usual description, since one can arbitrarily attach an odd parity to the charge. Therefore the result of the calculations is in agreement with the existing experimental evidence. The $\pi_{1}{ }^{0}$ of the isospin-triplet comes out as pseudo-scalar with respect to the parity of the second kind in agreement with the experiments. However, its parity of the first kind is even, like that of the charged pions.

The isosinglet state $\pi_{0}^{0}$ represents a pseudoscalar particle with respect to both definitions of parity. Its mass comes out as considerably higher than that of the $\pi_{1}{ }^{0}$ particles and 
it should therefore be highly unstable ${ }^{*}$. . Hitherto there seems to be no good experimental evidence for an isosinglet $\pi_{0}{ }^{0}$ particle.

The determination of the mass value leads to a satisfactory agreement between the first and the second approximation for the nucleons. Therefore, one may conclude that the correct mass value of the nucleons lies somewhere in the neighbourhood of $K \ell \sim 7$, which means that $\ell$ should be roughly equal to the Compton radius of the $\pi$ meson $\left(\ell \sim \frac{\hbar}{\mu_{\pi} c}\right)$, in order to yield the correct nucleon mass. For the $\pi$ mesons the two approximations give very different values. This unsatisfactory result seems to

TABLE I

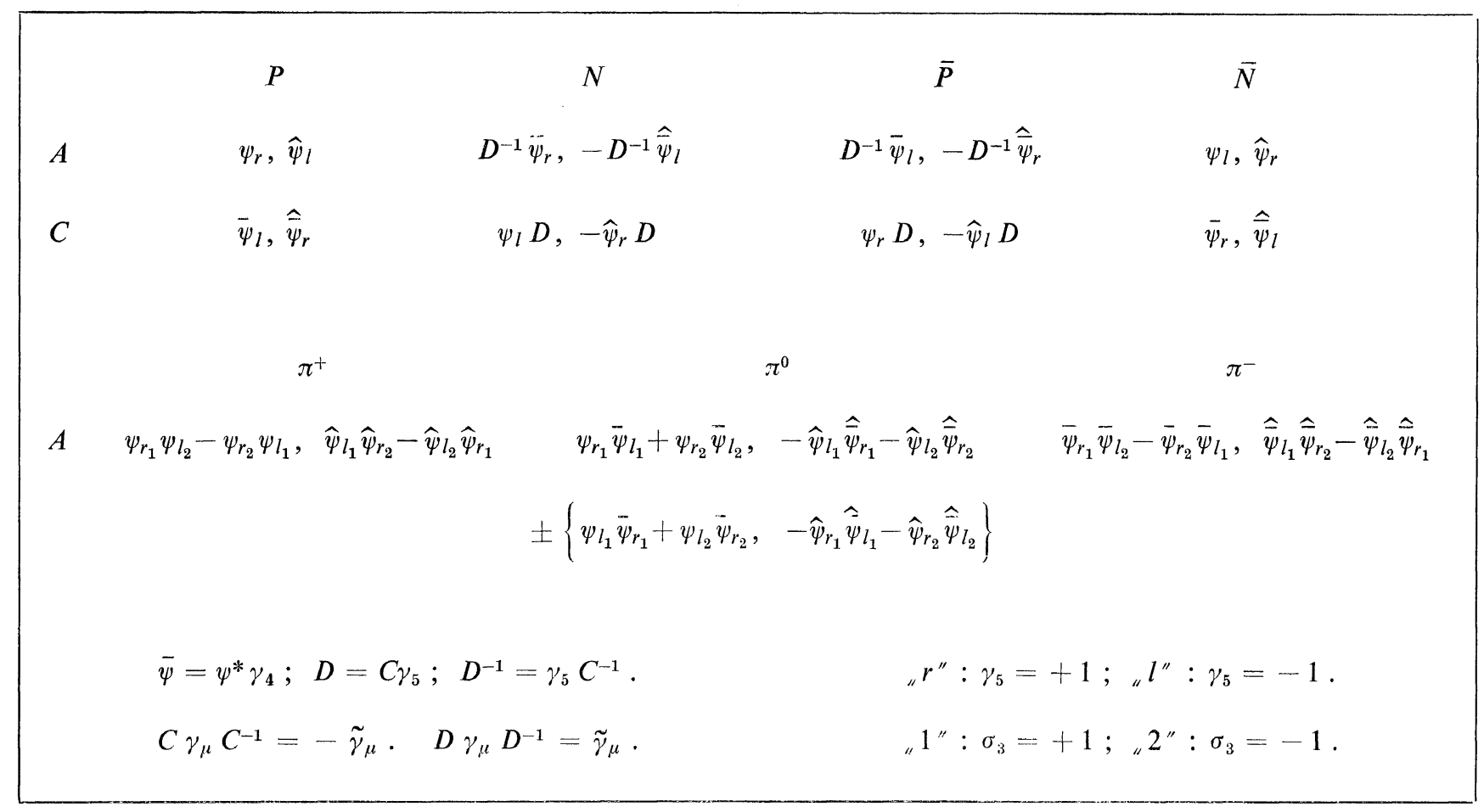

TABLE II

\begin{tabular}{|c|c|c|c|c|c|}
\hline \multirow{2}{*}{ Particles } & Nucleons & \multicolumn{2}{|c|}{$\pi$ mesons } & \multicolumn{2}{|c|}{$?$} \\
\hline & $\begin{array}{llll}P & N & \bar{P} & \bar{N}\end{array}$ & $\pi_{1}+\pi_{1}^{0} \pi_{1}^{-}$ & $\pi_{0}{ }^{0}$ & +0 & 0 \\
\hline $\begin{array}{l}\text { Parity } 1^{\text {st }} \text { kind } \\
\text { Partiy } 2^{\text {nd }} \text { kind }\end{array}$ & $\begin{array}{l}\text { not defined } \\
+--+\end{array}$ & $\begin{array}{l}++ \\
+\quad-+\end{array}$ & - & $\begin{array}{l}++ \\
+-\end{array}$ & $\begin{array}{l}- \\
-\end{array}$ \\
\hline Graph & & & & & \\
\hline $\begin{array}{ll}\text { Mass } K \ell & 1^{\text {st }} \text { approx. } \\
& 2^{\text {nd }} \text { approx. }\end{array}$ & $\begin{array}{l}7.08 \\
6.67\end{array}$ & $\begin{array}{l}3.0 \\
0.41\end{array}$ & $\begin{array}{l}7.0 \\
5.9\end{array}$ & - & $\overline{2.5}$ \\
\hline
\end{tabular}

(*) At the Conference the author had reported that the mass of the isosinglet state came out as equal to the mass of the isotriplet. A renewed controlling calculation has, however, revealed an error in the earlier computations of the neutral $\pi$-states, which did not affect the mass of the $\pi_{1}{ }^{0}$-state but did affect strongly the mass of the $\pi_{0}{ }^{0}$-state. The author regrets the error in his verbal report and wants to emphasize that an independent repetition of these rather complicated calculations by some other group - if possible by different methods - would be very desirable. 
be connected with the very high sensitivity of the meson mass in the calculations for changes in the nucleon mass. The $\pi$ eigenvalue depends on the nucleon mass and the calculation shows that a change of the nucleon mass by $10 \%$ produces a change of the meson mass by more than a factor 2. On the other hand, the nucleon mass can easily be inaccurate by $10-15 \%$. Therefore, a somewhat accurate determination of the $\pi$ meson mass has so far not been possible. But it may be possible in this special case to calculate later a genuine second Tamm-Dancoff approximation. The singlet $\pi_{0}{ }^{0}$ mass is not equally sensitive.

The wave equation (2) contains an indefinite sign in the non-linear term. A change of this sign is equivalent to a change of the sign of the commutator (3). For instance the transformation $x_{v} \rightarrow-x_{v}$ will change simultaneously the sign in (2) and in (3). But there is no simple transformation that leads from one sign in (2) to the other, without simultaneously also changing the sign in (3).

The only transformation that can produce this effect is a rather radical change made possible by the indefinite metric. If one changes the norm of all physical states of half-integer spin from +1 to -1 (keeping the norm of the states of integer spin as +1 ), one changes the sign of (3) without changing (2), and if one simultaneously replaces $x_{\nu}$ by $-x_{\nu}$, one changes the sign in (2) without changing (3).

By this transformation one gets into an entirely new set of states, which in relation to the first set can be considered as a non-combining term system. The assumption of a norm -1 of all states with half-integer spin does not inter- fere with the assumption of a unitary $S$-matrix. In this new system the particles corresponding to the nucleons get in the first approximation the same mass as those of the first system. The particles corresponding to the pions, however, get a different mass (the value is given in the third column in Table II). It is tempting to connect this non-combining term-system with the existence of the "strange particles" and especially the third column of Table II with the $\theta$-particles. But the analysis of the solutions has not yet been carried far enough to justify such an identification.

The mathematical analysis of the indefinite metric has not been carried further by the Göttingen group since the research connected with the Lee model. But I might mention at this point two recent papers by Ascoli and Minardi ${ }^{7}$, in which these authors try to get a more rigorous axiomatic basis for the use of the indefinite metric. One of their results concerns the equivalence between a local and causal theory with indefinite metric and non-local theory with definite metric. But I understand that Pauli will speak in the discussion about such problems, therefore I need not go into these questions.

In conclusion, I would like to state the present situation concerning the non-linear spinor theory as follows: the question whether this attempt can lead to a theory of the elementary particles is still completely open. But I think that the possibilities offered by the indefinite metric and the results reported here are interesting enough to make it worth while to look still more deeply into the consequences of such an attempt.

\section{LIST OF REFERENCES}

1. Heisenberg, W. Rev. mod. Phys., 29, p. 269, 1957.

2. Heisenberg, W. Nuclear Physics, 4, p. 532, 1957.

3. Heisenberg, W. and Pauli, W. On the isospin group in the theory of the elementary particles. (preprint)

4. Pauli, W. Nuov. Cim., 6, p. 204, 1957.

5. Gürsey, F. Relation of charge independance and baryon conservation to Pauli's transformations. (preprint)

6. Heisenberg, W. Nachr. Akad. Wiss. Göttingen, Math. phys. Kl. 2, p. 111, 1953.

Heisenberg, W. Kortel, F. and Mitter, H. Z. Naturforsch., 10a, p. 425, 1955.

7. Ascoli, R. and Minardi, E. On quantum theories with indefinite metric. (preprint)

\section{DISCUSSION}

Pauli: I am glad that during the last month I had occasion to discuss anew with many colleagues the problems of the elementary particles with respect to group theory and to field quantization. But the logical connection between field equations and commutation relations has not been sufficiently clarified neither by rigorous, nor by more clumsy intuitive methods. Therefore, reliable methods for calculating mass ratios of elementary particles are not yet available.
Regarding the papers of Heisenberg and collaborators on the spinor model, which appeared in the last years, after various discussions I reached the conclusion that they are mathematically objectionable.

To illustrate this, consider a spinor field $\psi(x)$ obeying a differential equation of first order

$$
\gamma^{v} \frac{\partial}{\partial x_{\nu}} \psi(x)+F(\psi(x))=0
$$


where the argument of the function $F$ depends only on the value of $\psi(x)$ at a particular point. (We assume that $F$ and its derivatives have no singularities; for example, imagine $F$ to be a polynomial.) Assume, further, that the field not only anticommutes for space-like points,

$$
\begin{gathered}
\left\{\psi_{\alpha} *(x), \psi_{\beta}\left(x^{\prime}\right)\right\}=0 \text { and }\left\{\psi_{\alpha}(x), \psi_{\beta}\left(x^{\prime}\right)\right\}=0 \\
\text { for }\left(x_{v}-x^{\prime}{ }_{v}\right)^{2}>0,
\end{gathered}
$$

but that this also holds for $x=x^{\prime}$

$$
\left\{\psi_{\alpha} *(x), \psi_{\beta}(x)\right\}=0
$$

where in the usual theory a singularity of the $\delta$-type appears.

As one knows from the Lee model, the commutation rules (2a) and ( $2 b$ ) can be fulfilled for an indefinite metric. They are, however, in contradiction to the assumption of a differential equation of the first order (Eq. (1)). Indeed, from (1) and $(2 a, b)$ it follows for all derivatives

$$
\left\{\psi_{\alpha}(x), \quad \frac{\partial^{n}}{\partial t^{\prime n}} \psi_{\beta}\left(x^{\prime}\right)\right\}=0 \quad \text { for } \quad t=t^{\prime} .
$$

This is in disagreement with the usually assumed mass spectra. The same contradiction still holds for several spinor fields obeying first order differential equations if the anti-commutativity of the field at the same point is supposed for all these fields.

Now the question arises whether in the quoted papers the contradicting assumptions $(1,2 \mathrm{a}, \mathrm{b})$ are actually used. In spite of the introduction of time-ordered products ( $\tau$ - and $\varphi$-functions) instead of the field operators themselves, it is my opinion that an assumption fully equivalent to the discussed one has been made in the course of the integration with the help of the Tamm - Dancoff method. Therefore, I do not trust the excuses which have been made for the contradicting results in question and I stress my opinion that the latter is still existing.

In this connection, I also wish to point out that I do not overlook the possibility of replacing the regularity of the anti-commutator at the same point by an oscillating behaviour with an essential singularity. This would give rise to an essentially new situation if this oscillating behaviour were explicity applied in the course of the integration of the equations for the $\tau$ - and $\varphi$-functions. But as soon as the oscillations are replaced by an average zero, which seems to me not admissible, the equivalence with the assumptions (1) $(2 a, b)$ and this contradiction reappears.

I disbelieve all the more in the possible excuses for such a contradiction as it can very easily be avoided. For instance, it is absent in the Lee model, where instead of (1) an integral equation appears. Indeed we know that the commutation relations (3) are not fulfilled in the Lee model with an indefinite metric.
My remarks do not pretend to say anything new. I only wanted to clarify my own position with regard to these problems. The general problem of whether an indefinite metric can be used in physics is not yet covered by my present remarks.

Heisenberg ${ }^{(*)}$ : Pauli's remark contains three different questions which I would like to answer separately and at some length in order to make this side of the theory completely clear. The three questions are :

1. Is the assumption that $\psi(x)$ and $\bar{\psi}\left(x^{\prime}\right)$ anticommute everywhere on the subspace $t-t^{\prime}=0$, but not for timelike $x-x^{\prime}$, compatible with the non-linear differential equation?

2. Are the assumptions made about the anticommutator used in a consistent way when they are applied in the Tamm-Dancoff method in connection with the equation $S(0)=S_{F}(0)=0$ ?

3. Should not the differential equation be replaced by an integral equation in the time-variable and is not the Lee-model an example for such a replacement?

For the first question we have to remember that - as had always been assumed - the anticommutator should behave near the light-cone like that classical solution of the non-linear differential equation that vanishes for spacelike distances. Such a solution has been constructed (the calculations had been carried out for the older spinor model, but not yet for the new wave equation) and it shows the following behaviour: it has infinitely frequent oscillations in the immediate neighbourhood of the lightcone for time-like distances. It vanishes for space-like distances. The statement that it vanishes everywhere for $t=t^{\prime}$ has, however, to be taken with some precaution: at the point $x=x^{\prime}$ its value is not defined, on account of the infinite oscillations. But its space integral at $t=t^{\prime}$ including the point $x=x^{\prime}$ definitely vanishes. There is no $\delta$-function of the space co-ordinates. Therefore, we see that this behaviour is actually compatible with the differential equation and there is no inconsistency in using an anticommutator of this (oscillating) type, which is, of course, not analytic at the light-cone.

With regard to the second question, we note that the non-linear wave equation of the operators can be considered as a compact way of writing the infinitely many differential equations between the $\tau$-functions of any number of variables. Actually, for the calculations it would be sufficient to assume that the field equation means nothing else but this infinite set of $\tau$-equations. (Instead of using the time-ordered products one could, of course, also use other kinds of products of field operators.) Now we know that the $\tau$-function

$$
\tau\left(x_{1} x_{2} \ldots \mid y_{1} y_{2} \ldots\right)
$$

behaves in the neighbourhood of the point, say $x_{i}-y_{k}=0$, like $\frac{1}{2} S_{F}\left(x_{i} y_{k}\right) \cdot \tau\left(x_{1} . x_{i-1}, x_{i+1} \ldots \mid y_{1} . y_{k-1} y_{k+1} \ldots\right)$ plus a

$\left(^{*}\right)$ This part of the discussion has not been reported verbatim but is based on a paper sent by Heisenberg after the Conference. 
function which is less singular near the light-cone. Therefore, the value of a $\tau$-function at the point $x_{i}=y_{k}$ is undefined in the same way as the value of $S_{F}(0)$.

On the other hand, those $\tau$-equations that result from the wave equation contain always one $\tau$-function (that with the higher number of variables) in which three coordinates are equal, e.g. either

$$
x_{i}=x_{k}=y_{l} \quad \text { or } \quad x_{i}=y_{k}=y_{l} \text {. }
$$

This is a necessary consequence of the postulate of microcausality. Therefore these $\tau$-equations have no definite meaning unless one defines explicitly what is meant in the equation by the value of the $\tau$-function at this point, e.g. $x_{i}=x_{k}=y_{l}$.

The simplest definition is given by the assumption that in the equations one shall put

$$
\begin{gathered}
\tau\left(x_{1} \ldots \mid y_{1} \ldots\right) \mid x_{i}=x_{k}=y_{l}=\lim _{\substack{x_{i} \rightarrow y_{l} \\
x_{k} \rightarrow y_{l}}}\left[\tau\left(x_{1} \ldots \mid y_{1} \ldots\right)\right. \\
\quad-\frac{1}{2} S_{F}\left(x_{i} y_{l}\right) \tau\left(x_{1} \ldots x_{i-1} x_{i+1} \ldots \mid y_{1} \ldots y_{l-1} y_{l+1} \ldots\right) \\
\left.-\frac{1}{2} S_{F}\left(x_{k} y_{l}\right) \tau\left(x_{i} \ldots x_{k-1} x_{k+1} \ldots \mid y_{1} \ldots y_{l-1} y_{l+1} \ldots\right)\right] .
\end{gathered}
$$

This limiting value exists on account of the behaviour of the $\tau$-functions and it gives a possible interpretation of the $\tau$-equations. The definition can be replaced by the simpler formula $S_{F}(0)=0$, or more accurately by the statement: " $\ldots$ in the infinite set of $\tau$-equations one can calculate as if $S_{F}(0)=0$ was generally correct". It is only this definition of the $\tau$-functions with three equal variables which gives a unique meaning to the wave equation. This meaning is compatible both with the oscillatory behaviour of the anticommutator and the postulate of microcausality. The equation $S_{F}(0)=0$ should be considered as a statement about the wave equation (or the $\tau$-equations) rather than as one about the anticommutator.

In momentum space our assumptions concerning the function $S\left(x x^{\prime}\right)$ or $S_{F}\left(x x^{\prime}\right)$ will probably mean the following. The mass spectrum $\varrho\left(K^{2}\right)$, from which $S\left(x x^{\prime}\right)$ can be constructed, will contain discrete states and a continuous part. For very large values of $K^{2}$ the continuous part will show an oscillating behaviour in order to represent the oscillating behaviour of $S\left(x x^{\prime}\right)$ near the light-cone. The discrete part will contain the discrete states like nucleons, electrons, etc. and the regularizing dipole ghosts. In the lowest approximations of the Tamm Dancoff method only the lowest discrete states and the dipole ghost can be taken into account; the higher discrete states and the continuum have to be neglected. Therefore, one obtains a reasonable approximation only if the contribution from the lowest states is considerably bigger than that of the higher ones and the continuum. This situation is well know $\mathrm{l}$ from the optical spectra of atoms. The conditions, that the lowest lines contain the main part of the total sum of $f$-values ( $\Sigma f_{v}=1$ is the Thomas - Kuhn sum rule) is frequently, but not always, fulfilled. The uncertainty of the Tamm - Dancoff method lies just in the uncertainty about the fulfilment of this condition.

The answer to the third question arises from the wellknown axiom of quantum field theory, according to which the field operators belonging to an arbitrarily small timeinterval $\Delta t$ must be sufficient to construct from vacuum the complete Hilbert space. Therefore, it must be possible to establish a unique relation between the operators referring to the interval between $t$ and $t+\Delta t$, and those belonging to the interval between $t+\Delta t$ and $t+2 \Delta t$. Since $\Delta t$ can be taken as arbitrarily small, this relation can be written as a differential equation in the time coordinate, which may possibly contain integrations over space co-ordinates.

However, it has to be noted that in a relativistic theory the occurrence of integrations over the space co-ordinates at this point would lead to a violation of the condition of microcausality. Even if the anticommutator vanishes for space-like distances within the time interval $t, t+\Delta t$, it would not fulfil this condition in the intervals between $t+\Delta t$ and $t+2 \Delta t$, if the connection would involve integrations over the space co-ordinates. Of course one could try to give up the condition of microcausality; but so far it has not been possible to drop this condition without violating at the same time the principle of macrocausality. It was one of the main results of the theory of special relativity, that causal connections have to be expressed as differential equations that are invariant for the Lorentz transformation. I cannot see how one could get away from this necessity.

Perhaps I should summarize the results of this discussion as follows : if one wants to keep microcausality, one has to connect the field operators at different times by means of a differential equation that is Lorentz invariant, or by other equations that are equivalent to such differential equations. The anticommutator between $\psi(x)$ and $\bar{\psi}\left(x^{\prime}\right)$ is zero for space-like distances and is indefinite at the point $x=x^{\prime}$; the space integral of the anticommutator between $\psi(x)$ and $\bar{\psi}\left(x^{\prime}\right)$ at the same time $t=t^{\prime}$ vanishes. Since the wave equation contains the product of three field operators at the same point one can give a definite meaning to the wave equation by defining this product or the corresponding $\tau$-functions by a limiting process, or more simply by $S(0)=S_{F}(0)=0$. Without such a definition the wave equation would not contain any statement at all.

Pauli: It is probably not true that the operators in one time-plane define the whole Hilbert space. You will have to take into account that $\psi_{\nu}$ is not a measurable field.

Heisenberg: That does not matter. It belongs to the Hilbert space; I mean, I can use any operators to define the Hilbert space, but I only need the assumption that the operators in a Hilbert space defined in a certain time-interval are sufficient to construct the complete Hilbert space, and I think that this assumption is completely true also in the Lee model. 


\section{Chew: I do not understand.}

Heisenberg: Oh yes! There is an apparent contradiction, because in the Lee model the renormalized wave equation takes the form of an integral equation in the time co-ordinate. In spite of this, it should be possible, on account of the axiom mentioned above, to reformulate it into a differential equation, which will probably involve integrations over space co-ordinates.

Pauli : I do not think we will reach an agreement on this point. There are many other points to discuss in Heisenberg's paper.

Stueckelberg: I just want to ask a rather trivial question. There is the $D_{C}$ (in Europe) or $D_{F}$ causality defined in terms of perturbation theory. Its basis is that we distinguish between positive and negative frequencies, and define $D_{C}$ or $D_{F}$ functions by positive frequencies. On the other hand, there is the causality, defined by dispersion relations, saying that the vacuum expectation value of the commutator $[\psi(x), \psi(y)]_{ \pm}$is only different from zero for time-like $x-y$. The question I am asking is whether Heisenberg or anybody in this group can answer the question: is there any relation between the condition of positive frequencies in the $D_{C},\left(D_{F}\right)$ definition and the positive energy equation in dispersion relations theory?

Oppenheimer: I think I can answer the question. If you have vanishing commutators, then you may construct either a causal or an anti-causal $D$-function. If you do not have vanishing commutators, it is very doubtful that you could construct either.

Gell-Mann: There is just one more comment in answer to Stueckelberg's question. If the causality in the sense of dispersion relations exists and you talk about the matrix elements of the commutators, then you can also talk, if you like, about the $D_{C}$ or $D_{F}$ functions for the same problem, and for positive frequencies in the Fourier transform these will agree and for negative frequencies they will disagree only in the sign of the imaginary part. So a statement about one is effectively a statement about the other, and the people working on the dispersion theories conventionally work with the commutator rather than with the $D_{C}$ and $D_{F}$ function just for greater convenience.

Stueckelberg: Does this mean that if you speak of commutators you do not really need somehow a series development?

Gell-Mann: Well, one hopes in dispersion theory not to make use of perturbation theory. Of course, nobody really knows how to work with field theory aside from perturbation theory but people try, and they have produced a certain number of results especially in dispersion theory.

Oppenheimer: The answer is the following. Of course, to know the commutators in full you need to know the theory and know whether it is linear or not, but to know that something vanishes will be true of all functions. Thus this statement does not restrict you to any linear theory.

Klein: I would like to ask a very simple question which can be actually answered. Will Heisenberg please remind us of what is the meaning of the graphs for the calculations of the masses.

Heisenberg: When you work with the Tamm - Dancoff approximation you deal with the $\tau$-function $\langle\Omega|\psi(x)| \Phi\rangle$. Then from this $\tau$-function you go over to some function with more variables, by means of a Green's function, using the wave equation which has been written. So this is something like, say

$\langle\Omega|\psi(x)| \Phi\rangle=\left\langle\Omega\left|d x^{\prime} G\left(x, x^{\prime}\right) \psi\left(x^{\prime}\right) \bar{\psi}\left(x^{\prime}\right) \psi\left(x^{\prime}\right)\right| \Phi\right\rangle$.

Then you can again go over to the next step and you introduce two Green's functions,

$$
G\left(x, x^{\prime}\right) \cdot G\left(x^{\prime}, x^{\prime \prime}\right) .
$$

In this way you get higher $\tau$-functions and then at the end you make the so-called contractions. Thereby these $\tau$-functions can be expressed approximately by the $\tau$-functions of a lower number of variables. The contraction function is essentially the commutator, or more accurately the Schwinger $S_{F}$-function. The graph states which points $x$ or $x^{\prime}$ or $x^{\prime \prime}$ are to be connected by $G_{F^{-}}$or $S_{F^{-}}$-functions in the final integral equation.

Thereby one can get a simple formal picture for such a rather complicated integral equation, just as in the conventional Feynman-graphs.

Johnston: I should like to ask Heisenberg one point about the masses of the $\pi_{0}{ }^{0}$ and $\pi_{1}{ }^{0}$ in the scheme he drew up on the board. He said that in the first approximation the masses became equal, and he said that perhaps if other corrections were included, mentioning particularly electromagnetic corrections, this situation would possibly change. Does the electromagnetic interaction come from the first wave equation written on the board, or is this something extra that has to be added?

Heisenberg: That part of the calculation concerning the electromagnetic fields and particles has not yet been carried out for this wave equation, but it had, in a former paper by Ascoli and myself, been done for the old nonlinear spinor equation. At that time it turned out that the electromagnetic forces can come out of such an equation; actually they are contained in it. So at this point we do not yet have any definite results for the new equation, but I think it is very probable that one can just repeat the old calculations for this model here and we will get very similar results.

Wentzel: There are so many questions to be asked that I can hardly start, but let me ask what has become of the problem that you once connected with the degeneracy of the vacuum; the necessity to explain the large number of quantum numbers one needs, for instance, in baryon physics 
Heisenberg: I think this question of a duplication of the vacuum is more a matter of calculation technique than of fundamentals. For instance, when we have the solutions for the leptons and for the baryons then, of course, we can separate them in two ways; one can either say that the operator $\psi$ is the sum of two operators, one referring to changes of the baryonic number, and the other referring to changes of the leptonic number, but one can also introduce two kinds of vacuum for the matrix element between a state and the vacuum, so that instead of doubling the operators we double the vacuum, and thereby one can perform transformations not only on the operators but also on the vacuum. If one has to calculate a vacuum expectation value of a product of not only two but $2 n \psi$-functions, then, of course, this value would become a matrix with $2^{n}$ rows and columns. But perhaps I should state it more clearly in the following way. In the old theory of the atom, when Dirac introduced the spin, his argument was as follows. In the normal theory one has momentum and co-ordinate $p$ and $q$ and we get an equation of the second order on account of the Lorentzinvariance. But now, instead of taking a square root, one can also introduce a new degree of freedom which can be put on the wave functions, which means on the Hilbert vectors. In this way, Dirac introduced the duplication of states in Hilbert space by saying: we have not only one state vector, namely a function $\psi$, but we have $\psi_{1}$ and $\psi_{2}$ and thereby we have a duplication of the states. In the same way you can say here that we start from a Lagrangian in which, of course, there is nothing to be seen of any duplication; but when we now try to find a commutator which corresponds to this Lagrangian, it may turn out that a square root is to be taken (e.g. as for the mass in the Klein-Gordon-equation), and therefore a duplication may occur in the vacuum or in the state vectors.

Wentzel: This partly answers my question, but looking at reality we have a quantum number that is called strangeness. Is the multiplicity that is inherent in your formalism sufficient to explain or to incorporate all the quantum numbers which we need nowadays? Of course you can go on and introduce a $\sigma$ and a $\Sigma_{1}$, and by always taking another power of two you can get there. But is this a natural procedure?

Heisenberg: I think it is characteristic for these quantum numbers - you referred to the quantum numbers called $l$ and $l_{N}$ in the preprint - that they are apparently defined only by cyclic groups. We need no space group, no rotation in space, to explain these quantum numbers. It seemed natural to us that just the cyclic groups should be put into the Hilbert space. It was especially emphasized by Dürr that actually cyclic groups may be transferred to the vacuum while space rotations can not. The reason is the following: if the operators transform like spinors, then if one tries to replace their transformation by transformations in Hilbert space, $\Lambda^{\prime}=T^{-1} A T$, one needs for the corresponding transformations in Hilbert space not half-integral representations of the rotation group but quarter-integral representations which do not exist. Therefore, three-dimensional rotations can certainly not be put on the vacuum, while one-dimensional rotations can be transferred on the Hilbert space. It seemed natural to connect this result with the other empirical fact that these extra groups are actually only cyclic groups. So I think it is not unnatural to say that these two cyclic groups belonging to $l$ and $l_{N}$ are actually properties of the operators which can be expressed by a duplication of the vacuum or of the state vectors.

Pauli: I completely disagree with the answer of Heisenberg. I think this is not only unnatural but this is mathematically impossible. You cannot use the multiplicity of the vacuum in order to obtain the strangeness, because then the charge of two protons is not any longer two, if the charge of one proton is one. This I discussed already in April and I wonder that you again repeat it all.

Heisenberg: Of course I again disagree completely with what Pauli said, because I do not see the slightest reason why one should not take out of the vacuum as many charges as one has protons; that is the same as in the Dirac theory. Dirac has introcuded the doubling for each new electron, and for ten electrons he had also ten spins.

Pauli: The point is that the additivity of charges is different from the additivity of other operators, so that the answer was again completely false: the additivity of charges is different from the additive behaviour of other operators like spin and isotopic spins, and masses. This is the point. The number of the vacua does not multiply if you have two protons. I think this was disproved in April.

Gell-Mann: May I attempt to re-state the situation? I am not sure if I understand all points but perhaps it will come out. As I understand the idea, it would be something like this. You take a twin vacuum and an operator, which you would ordinarily call a neutron operator. You act on the twin vacuum with the neutron operator. On one state it makes a neutron and on another state it makes a $\Lambda$. So you get two states, neutron and 1. I think the difficulty is, that if you take two neutron operators, the two vacua will give you only double the number of states but you need four times as many states now, namely neutron-neutron, neutron- $\Lambda, \Lambda$-neutron, and $A-\Lambda$. So, depending on how many particles you want to describe, $N$, you need $2^{N}$ vacua. This must vary with the number of particles we wish to describe. I suppose one can do it but it seems to be complicated and very much like adding another field.

Heisenberg: I agree completely with what Gell-Mann just said about this point; but at the same time, I propose to postpone the discussion for half a year and then we will know more about it.

Pauli: Well, I think that it is superfluous. I think that in half a year the answer would be the same as Gell-Mann gave just now. 